\title{
Frontline clinician concerns during the COVID-19 pandemic: A qualitative inquiry
}

\author{
Rachel Vanderkruik*1,2, Helen R. Mizrach ${ }^{1,3}$, Sydney Crute ${ }^{1,3}$, Cayley C. Bliss ${ }^{3}$, Louisa G. Sylvia ${ }^{1,4}$, Lara Traeger ${ }^{1,2}$, \\ Daniel L. Hall ${ }^{1,3}$, Christina M. Luberto ${ }^{1,3}$, Joanna M. Streck ${ }^{1,2}$, Amelia M. Stanton ${ }^{1,2}$, Nevita George ${ }^{1,4}$, Sara E. \\ Looby $^{1,5}$, Darshan H. Mehta ${ }^{1,5}$, Gregory L. Fricchione ${ }^{1,5}$, Elyse R. Park ${ }^{1,3}$ \\ ${ }^{1}$ Massachusetts Hospital/Harvard Medical School, Boston, MA, United States \\ ${ }^{2}$ Department of Psychiatry, Massachusetts General Hospital, Boston, MA, United States \\ ${ }^{3}$ Health Policy Research Center, Mongan Institute, Massachusetts General Hospital, Boston, MA, United States \\ ${ }^{4}$ Dauten Family Center for Bipolar Treatment Innovation, Massachusetts General Hospital, Boston, MA, United States \\ ${ }^{5}$ Benson-Henry Institute for Mind Body Medicine, Massachusetts General Hospital, Boston, MA, United States
}

Received: February 22, 2021

DOI: $10.5430 /$ jha.v10n2p21
Accepted: March 24, 2021

Online Published: April 14, 2021

URL: https://doi.org/10.5430/jha.v10n2p21

\begin{abstract}
Objective: The COVID-19 pandemic has strained healthcare systems worldwide, placing a high psychological burden on frontline clinicians. There is an urgent need to better understand their stressors and determine if stressors differ by clinical role. The present study assessed the concerns among frontline clinicians across a large healthcare system during the COVID-19 pandemic to inform the development of tailored supportive services.

Methods: From March - June 2020, frontline clinicians across the Mass General Brigham healthcare system were invited to register for an adapted mind-body resiliency group program. Clinicians completed pre- and post-program assessments asking them to report their COVID-19-related concerns. Qualitative data were analyzed in aggregate and by clinical role using content analysis to identify overarching domains.

Results: Frontline clinicians' concerns fall within seven domains: concerns for self, patients, family members, staff, existential concerns, systems-level concerns, and job-level concerns. Concerns for self and existential concerns were most commonly reported across clinical roles. Long-term care clinicians were highly concerned about patients' wellbeing while rehabilitation therapists were highly concerned about their family members' health. Across groups, nurse practitioners and physician assistants more often reported job-level concerns. Concerns for staff and systems level concerns were less frequently reported across clinical roles.

Conclusions: Frontline clinicians share common pandemic-related concerns, but nuances exist among the concerns most frequently reported across clinical roles. Interventions that offer stress management and resiliency training may be helpful for addressing pandemic-related concerns overall. Future research should determine if tailored support services by clinical role may be warranted.
\end{abstract}

Key Words: COVID-19 concerns, Frontline clinician, Pandemic concerns, Coronavirus, Stressors, Resilience

*Correspondence: Rachel Vanderkruik; Email: rvanderkruik@mgh.harvard.edu; Address: Massachusetts General Hospital/Harvard Medical School, 185 Cambridge Street Boston, MA 02114, United States. 


\section{INTRODUCTION}

The Coronavirus (COVID-19) pandemic has put a significant strain on healthcare systems around the world. Hospitals and their staff members face unprecedented challenges to ensure that they have the space, resources, and personnel available to care for patients while also trying to limit the spread of the virus. Frontline healthcare clinicians include physicians, nurses, advanced practice clinicians, rehabilitation therapists, mental health clinicians, and others involved in the diagnosis, treatment, and care of patients with COVID19. Changing clinical guidelines, lack of personal protective equipment, longer work hours, lack of adequate support, and constant pandemic-related media coverage may all contribute to the burden placed on frontline clinicians. ${ }^{[1-4]}$ Additionally, health-related uncertainty and fatigue caused by the pandemic within hospital systems and across all sectors of society can induce stress. ${ }^{[2,5]}$ Frontline clinicians reported an elevated prevalence of depression, anxiety, insomnia, burnout, post-traumatic stress symptoms, and distress associated with the COVID-19 pandemic. $^{[1,4,6-8]}$ This psychological burden impacting frontline clinicians could have detrimental implications, such as a reduced ability to care for themselves, their patients, and their families.

Frontline clinicians are at an elevated risk of developing mental health symptoms when compared to their colleagues not serving on the front lines. ${ }^{[1,7]}$ In particular nurses and advanced practice clinicians may have signs of heightened COVID-19 related distress as compared to frontline clinicians serving other clinical roles. ${ }^{[1,8-10]}$ However, few studies have reported on whether pandemic-related concerns differ by clinical role. Khanam et al. ${ }^{[10]}$ surveyed frontline healthcare clinicians through an online semi-structured questionnaire and found that feeling sad and pessimistic, feeling of being avoided by others, the burden of change in the quality of work (e.g., insufficient PPE, lack of staff), and worrying whether family will be cared for in their absence were reported significantly more often among nurses than among physicians. Other studies have identified COVID-19 related concerns faced by surgical staff, nurses, and other healthcare clinicians but have not directly compared concerns between clinical roles. ${ }^{[10-12]}$

During the ongoing COVID-19 pandemic, it is critical for hospital systems to offer support services to frontline clinicians. A narrative review of 96 articles addressing clinician mental health since the start of the current pandemic identified the need for resilience and stress reduction training, social support interventions, and normalization and provision of mental health support programs. ${ }^{[13]}$ Although strategies have been proposed to decrease psychological burden and improve resilience among frontline clinicians, such as peer support interventions ${ }^{[14]}$ or digital support packages, ${ }^{[15]}$ few interventions have been implemented or analyzed. ${ }^{[13,16]}$ The need to directly consult with frontline healthcare clinicians to understand their stressors and tailor interventions accordingly has been identified. ${ }^{[3,14,15]}$ Obtaining insight into pandemicrelated concerns that are most central to frontline clinicians is essential to adequately inform the development of future support systems and interventions.

In response to the pandemic, the Massachusetts General Hospital (MGH) Psychiatry Department recognized the need for system-wide services to promote resiliency and stress coping ability among frontline clinicians. We adapted an evidencebased resiliency program to meet the perceived needs of frontline healthcare clinicians across a hospital system during the onset of the pandemic. The present study aimed to better understand the concerns among frontline clinicians during the COVID-19 pandemic to determine what types of services and program content may be most helpful moving forward. We also explored how pandemic-related concerns differed by clinical role.

\section{Methods}

This study was conducted at the MassGeneral Brigham (MGB) umbrella healthcare system in Boston, Massachusetts, US. The study protocol was approved by the MGB Institutional Review Board and a COVID-19 internal board review.

\subsection{Study design}

English-speaking clinicians $(\mathrm{n}=102)$ across the MGB healthcare system who cared for patients during the COVID-19 pandemic registered for the resiliency groups, between 03/23/20$06 / 02 / 20$, and completed brief pre and post-group treatment questionnaires via Research Electronic Data Capture (REDCap). The mind-body resiliency group content was modified to address perceived stressors faced by frontline clinicians during the onset of the pandemic. ${ }^{[17]}$ Groups were conducted via Zoom twice weekly for 4 weeks, and participants were assigned to a group based on their clinical role. Significant improvements were reported in resiliency, stress coping, emotional distress, loneliness, mindfulness, and self-compassion. ${ }^{[18]}$

\subsection{Sociodemographic information}

Demographic information collected included age, sex, race and ethnicity, and clinical role.

\subsection{Frontline clinician concerns}

In order to capture any concerns during the pandemic, participants were asked the following question in an open-text survey: "Thinking about your work in the COVID-19 pan- 
demic, what concerns you the most at this time?" at their baseline and post-treatment assessment.

\subsection{Data analysis}

Two trained Research Coordinators (Authors: SC and CB) coded all data independently using NVivo 11. Data were collapsed across both assessment timepoints to report on all concerns reported by participants early in the pandemic, over the timeframe of March to June 2020. We did not report on differences in themes between pre- and post-intervention as the intervention was designed to provide coping skills for stress and was not designed to ameliorate objective stressors. Data were analyzed using content analysis to identify major concepts and axial coding to group and connect data into meaningful categories. ${ }^{[19]}$ The analysis followed Braun and Clarke's six phases of analysis: (a) familiarization: reading and rereading the data, noting down initial ideas, (b) generating initial codes: systematically coding interesting features of the data across the entire data set and collating data relevant to each code, (c) searching for themes: collating codes into potential themes, (d) reviewing themes: checking the themes in relation to the coded extracts and the entire data set, (e) defining and naming themes: refining the specifics of each theme to generate clear definitions and names, and (f) producing the report: selecting extract examples, relating the analysis back to the research question. ${ }^{[20]}$ At each analysis phase, the coders and the principal investigator (Author: EP) compared their results to confirm consistency, resolving discrepancies through discussion and comparison of the raw data. Themes within each content area were identified, and responses were categorized into codes. The coders then refined their definitions and the content of the codes and compared their coding lists. The final reliability score (Cohen's kappa) was 0.92 suggesting high inter-coder agreement.

Analyses were conducted in aggregate and stratified by clinical role. For the stratified analyses, we decided to not include three of the clinician groups (advance practice clinicians, technicians/translators, and "other" clinicians) due to the small sample size of participants in each group $(n=3-4$ per group), which would limit our ability to rank responses within these groups. We first grouped clinician responses together by the thematic domain within each of the remaining five clinician groups. We then ranked each of the seven overarching thematic domains by the frequency they were reported by participants within each of the remaining five clinical role groups.

\section{ReSults}

\subsection{Participants}

As reported in Park et al., ${ }^{[18]}$ program participants had a mean age of 45 years, were $92.1 \%$ female, $83.3 \%$ white, from 15 institutions, and had varied clinical roles (see Table $1)$.

\subsection{Concerns about the COVID-19 pandemic}

There were seven overarching themes that emerged regarding clinicians' concerns, as presented in Table 2.

Table 1. Frontline clinician characteristics $(n=102)$

\begin{tabular}{ll}
\hline Characteristic & $\mathbf{N}(\mathbf{\%})$ \\
\hline Age (M, SD) & $45.1(12.2)$ \\
Female Sex & $93(92.1 \%)$ \\
Race/Ethnicity & \\
White, non-Hispanic & $85(83.3 \%)$ \\
Black & $4(3.9 \%)$ \\
Hispanic & $10(9.8 \%)$ \\
Asian & $9(8.8 \%)$ \\
Other & $2(2.0 \%)$ \\
Clinical Role & \\
Physicians (MDs) & $13(12.8 \%)$ \\
Nurse Practitioners (NPs) / Physician Assistants (PAs) & $16(15.7 \%)$ \\
Registered Nurses (RNs) & $18(17.7 \%)$ \\
Advance Practice Clinicians (Midwives / Nurse Anesthetists) & $3(2.9 \%)$ \\
Occupational/ Physical/ Respiratory/ Speech/Diet Therapists & $19(18.6 \%)$ \\
Social Workers (SWs) / Chaplains / Psychologists & $25(24.5 \%)$ \\
Technicians / Translators & $4(3.9 \%)$ \\
Other & $4(3.9 \%)$ \\
\hline
\end{tabular}


Table 2. Frontline Clinician concerns regarding work in the COVID 19 pandemic

\begin{tabular}{l}
\hline Thematic Domain \\
\hline 1. Concerns for self (e.g., physical and emotional wellbeing) \\
2. Concerns for patients (e.g., safety and receipt of adequate care) \\
3. Concerns for family members (e.g., spreading virus to them, reduced time with them) \\
4. Concerns for staff (e.g., safety and strain on social dynamics) \\
5. Existential concerns/concerns for state of world (e.g., uncertainty about disease and impact on society) \\
6. Systems-level work concerns (e.g., lack of leadership or supplies) \\
7. Job-level concerns (e.g., impact on job stability or roles)
\end{tabular}

\subsubsection{Concerns for self}

Among concerns for self, the most commonly reported subtheme was concern about one's own physical health (e.g., getting sick, dying, inability to pay for medical care). For example, one participant expressed concerns about "getting sick at work, not being able to afford medical care, becoming seriously ill and dying from going to work..." Anticipated challenges associated with experiencing difficult emotions (e.g., fear, distress, loneliness) was another common concern. For example, one participant wrote, "managing my own stress properly; how can I help my patients if I don't always feel okay? [I] don't seem to have very good coping skills..." Other concerns included feelings of hopelessness or helplessness, questioning their career (e.g., no longer enjoying their job), and being poorly treated by their patients or family members.

\subsubsection{Concerns for patients}

Among concerns for patients, providing adequate care and maintaining patient safety were the most common concerns. For example, one participant expressed concerns about the "ability to maintain a level of care that these patients deserve...not being able to comfort patients and families..." The second most commonly reported theme pertained to safety concerns, such as, "constantly feeling as though I am exposing my vulnerable patients." Other less commonly reported subthemes included limitations of telehealth (e.g., sustainability of virtual visits, difficulty with connecting virtually), how to ensure quality care for patients, concerns regarding future patient interactions, limited social support (e.g., patients isolated from loved ones), limited engagement in care (e.g., medical decision making), and limited patient-provider relationships (e.g., not enough time to connect with patients).

\subsubsection{Concerns for family members}

The most commonly reported subthemes in this category were concern for family members' health and lack of time with family and friends. One participant wrote, "my single largest fear is bringing home the illness and my husband or parents dying from it." In regards to lack of time, a partici- pant expressed "concern about being able to see family from afar, and that it has been a very long time. Hope to see them soon, missing people." Other less frequently reported subthemes included limited resources, especially childcare (e.g., lack of daycare, schools, and/or camps), and ability to care for family members (e.g., elderly or sick family members).

\subsubsection{Concerns for staff}

Among concerns for staff, the most commonly reported subthemes were maintaining staff safety (e.g., "I have had no symptoms, but would hate to be a silent carrier that could infect others in my work setting") and impact on relationships (e.g., tense and stressful co-worker relationships, inability to support colleagues). Some clinicians identified concerns regarding the ability to maintain staff emotional well-being and having sufficient staff to do necessary jobs and tasks over the course of the pandemic.

\subsubsection{Existential concerns/concerns for state of world}

With respect to existential concerns/concerns for state of the world, participants were most distressed by the uncertainty surrounding the disease (e.g., "uncertainty about COVID....lack of knowledge [about] this novel virus" ) and potential changes to society (e.g., "uncertainty around when this will go back to our new normal"). General worry for the well-being of others was also commonly reported. For example, one participant reflected on concern for "the collective trauma inflicted and long-term consequences of that." Other less commonly reported subthemes included concerns for vulnerable populations, the sustainability of the workforce, and the future of healthcare and hospital dynamics.

\subsubsection{Systems-level work concerns}

Among systems level work concerns, the most commonly reported subtheme was the lack of leadership or oversight of guidelines. As one participant reported, "I am concerned that I cannot trust our national and hospital guidelines on how to protect ourselves, that the guidelines are written based on PPE available, not based on what is safest for us. I'm concerned that we are routinely in unsafe situations." Participants also reported concern regarding the lack of supplies 
(e.g., "Continued limited supplies of PPE and medical supplies"). Other less common subthemes included the lack of (or inaccurate) COVID-19 testing for patients and staff, concerns regarding procedures around returning to the office, challenges with work from home, and maintaining workflow procedures.

\subsubsection{Job-level concerns}

Participants were most concerned about the future status of their careers. For example, one participant wrote, "my concerns right now are how will the COVID-19 pandemic change my role now and in the future, will my work be different from now on and how will I cope with that." Participants also expressed concerns regarding job stability (e.g., "future job security due to economic downfall"). Other less commonly reported subthemes included concerns about workload, scheduling, and redeployment (e.g., mandatory shifting of job and/or schedule within the hospital).

\subsection{Themes by clinical role}

When assessing concerns across different groups of clinicians, concerns for self, including one's physical health and emotional wellbeing, was a top concern across all clinical roles. Additionally, existential concerns regarding the state of the world and uncertainty for the impact of the pandemic on the future of society was a common theme across all clinical groups. However, the degree to which the seven themes above were reported varied within each clinical role. Nurses, social workers, and psychologists were highly concerned about patients' health and wellbeing. Rehabilitation therapists (i.e., physical therapists, occupational therapists, and respiratory therapists) commonly reported concerns for their family members' health and wellbeing. Of the clinical groups, nurse practitioners/ physician assistants more often reported career-level concerns, such as job security or changes to their roles. Concerns for staff and systems level work concerns were less frequently reported across all clinical groups. See Figure 1 below for a graphical overview of how commonly the themes regarding pandemic concerns identified in Table 1 above were reported across the five clinician groups.

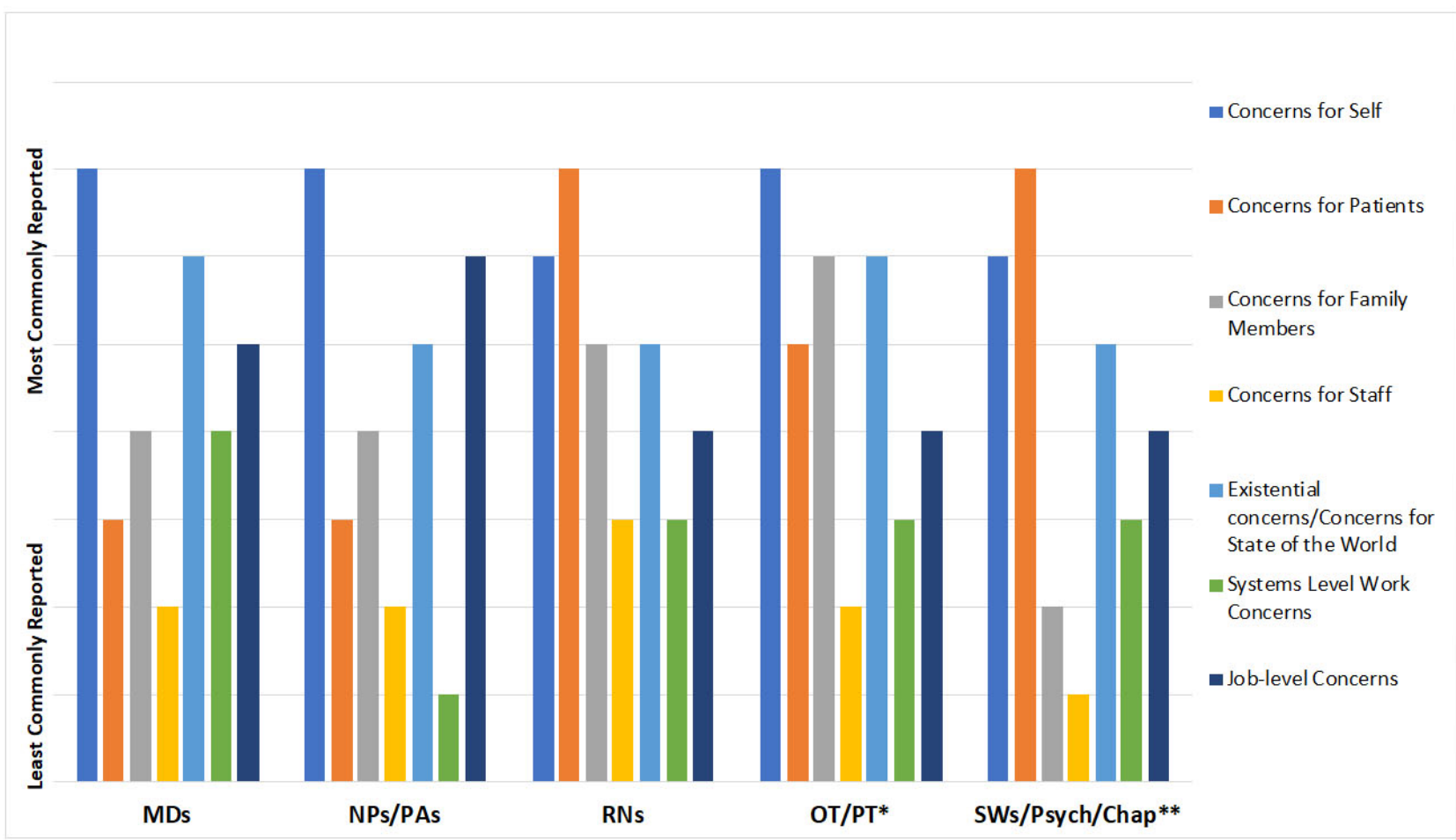

Figure 1. Commonality of thematic concerns by clinical role *Occupational/Physical/Respiratory/Speech/Diet Therapists; **Social Workers (SWs)/Psychologists/Chaplains. The length of the bars correspond to the frequency that each of the seven thematic concern domains were reported by clinicians within each clinical role group. The longest bars correspond to the thematic domain most commonly reported, while the shortest bars correspond to the thematic domain least commonly reported. 


\section{Discussion}

The COVID-19 pandemic has imposed an unprecedented burden on healthcare systems and frontline clinicians. This study sought to (1) explore common pandemic-related concerns among frontline clinicians who participated in a stress management and resilience program and (2) assess the degree to which these concerns differed by clinical role. Our findings suggest that frontline clinicians' concerns fall within seven key areas: concerns for self, concerns for patients, concerns for family members, concerns for staff, existential concerns/concerns for the state of the world, systems-level work concerns, and career level concerns. The seven areas identified align with the concerns previously reported in the literature from frontline medical workers across healthcare systems and countries. ${ }^{[3,9-11]}$ These concerns may be contributing to the psychological toll of the pandemic on clinicians around the world ${ }^{[1,4,6-8]}$ and may result in increased burnout, decreased productivity, and more adverse outcomes for patients. A review of best practices for addressing the mental health needs of frontline health workers during pandemics identified minimal interventions that have already been implemented and concluded that evidence-based interventions are urgently needed to better serve health workers both during the current pandemic and in future pandemics. ${ }^{[21]}$ Interventions that specifically address their concerns rather than teaching generic approaches to stress reduction and resilience may be warranted. ${ }^{[3]}$ Our findings may inform the development of services and interventions offered to clinicians by ensuring that common concerns are addressed. Increased awareness of these concerns may also be helpful for therapists or mental health providers who are working with frontline clinicians during this time, in that they may be more able to normalize, validate, and address such concerns as appropriate.

To our knowledge, this study is one of the only analyses that examines the degree to which pandemic-related concerns vary by clinical role. While we did observe some differences in commonly reported themes by clinical role, we also observed commonalities. Notably, concerns for self (e.g., physical and emotional wellbeing) were commonly reported across all groups. Some of the differences that we noticed in the reporting of certain themes across clinician type may be due to the nature of the work and responsibilities for a given role. For example, we found that nurses, social workers, and psychologists were highly concerned about patients' health and wellbeing, possibly because they are clinicians who are typically involved in the long-term support and care of patients. While our findings suggest that all clinicians share common concerns in response to the pandemic, our findings suggest that there may be some differences by clinician type.
Future research could further explore nuances across clinical roles that could determine when or if tailored support services by clinical role are warranted.

The findings of this study should be interpreted with several limitations in mind. The question assessing clinicians' concerns was an open text field, and we did not probe or follow-up with participants to capture a comprehensive list of concerns. As the pandemic stressors were rapidly evolving throughout the months of study enrollment, there may be additional concerns that were not captured by this one question, and thus we purposefully did not try to capture changes in concerns. Additionally, the number of participants within each clinical role ranged from 3 to 25; these sample sizes limit our ability to compare the frequency of themes across groups as well as our ability to draw concrete conclusions regarding differences in concerns across groups. We did not include the analyses from advance practice clinicians, technicians/translators or "other" participants in this portion of the analyses due to having too small of a sample size within these groups to make generalizable comparisons. They were also the only clinical groups where no providers had reported some of the thematic domains, which was likely due to having fewer opportunities to generate sufficient diversity in responses. Furthermore, this study was limited to English speaking participants and we did not assess for the economic status of participants, which further limits our knowledge of how generalizable these findings are to the broader frontline clinician population.

Despite these limitations, this study contributes meaningful and timely insight into the nature of the concerns among frontline clinicians during a pandemic. Data collection for the study started in April 2020, which was relatively soon after the pandemic began to take a toll on the MGB healthcare system. Given that the effects of the COVID-19 pandemic continue to change and evolve, it would be of interest to further assess how concerns differ from the start of the pandemic to later timepoints. Additionally, a strength of this study is the robust sample size and rigorous qualitative data analysis. The inclusivity of clinicians from many different roles and specialty groups allowed for the identification of common concerns that are role specific and across all clinical roles.

A mixed methods systematic review concluded that there is a lack of both quantitative and qualitative evidence from studies conducted during pandemics that can inform the selection of interventions that may increase the resilience and improve the mental health of frontline providers. ${ }^{[22]}$ This review also suggested that interventions should assess and address healthcare providers' specific concerns. When designing such interventions, it may be helpful to consider 
whether or not specific concerns can be directly addressed through system changes or support services. For concerns that can be directly addressed (e.g., PPE availability, system level concerns), future research could explore what types of support or changes would alleviate these concerns. For concerns that cannot be changed at the system-level (e.g., uncertainty about the world or future), interventions that offer stress management and resiliency training may be most helpful. Our findings indicate that it may be beneficial to have different types of interventions targeting different levels, such as at the provider-level or the healthcare system-level. This study contributes to the identified gap in knowledge regarding pandemic-related concerns among frontline clinicians and may inform the development of interventions designed to address such concerns and support the mental health and wellbeing of healthcare providers.

\section{ACKNOWLEDGements}

We would like to thank all the clinicians and MGH Psychiatry leadership for volunteering their time and support at the onset of the pandemic to support the needs of frontline clinicians. We would like to thank the MGH Benson-Henry Institute for Mind Body Medicine, Home Base, and the Mongan Institute for provision of support for this study.

\section{FUNDING/SUPPORT}

None to report. We would like to express our gratitude for our team who volunteered their time, at the onset of the pandemic, to assist frontline clinicians.

\section{DATA AVAILABILITY STATEMENT}

The datasets during and/or analyzed during the current study available from the corresponding author on reasonable request

\section{CONFLICTS OF INTEREST Disclosure}

Dr. Sylvia receives royalties from New Harbinger and has been funded by NIH, Takeda, PCORI, and AFSP in the past 36 months. All other authors declare that they have no competing interests.

\section{REFERENCES}

[1] Lai J, Ma S, Wang Y, et al. Factors Associated With Mental Health Outcomes Among Health Care Workers Exposed to Coronavirus Disease 2019. JAMA Network Open. 2020; 3(3): e203976-e. PMid: 32202646. https://doi.org/10.1001/jamanetworkopen. 20 20.3976

[2] Tomlin J, Dalgleish-Warburton B, Lamph G. Psychosocial Support for Healthcare Workers During the COVID-19 Pandemic. Front Psychol. 2020; 11: 1960. PMid: 32849149. https://doi.org/10.3 389/fpsyg. 2020.01960

[3] Shanafelt T, Ripp J, Trockel M. Understanding and Addressing Sources of Anxiety Among Health Care Professionals During the COVID-19 Pandemic. JAMA. 2020; 323(21): 2133-4. PMid: 32259193. https://doi.org/10.1001/jama.2020.5893

[4] Geoffroy PA, Le Goanvic V, Sabbagh O, et al. Psychological Support System for Hospital Workers During the Covid-19 Outbreak: Rapid Design and Implementation of the Covid-Psy Hotline. Front Psychiatry. 2020; 11: 511. PMid: 32670100. https://doi.org/10.338 9/fpsyt.2020.00511

[5] El-Hage W, Hingray C, Lemogne C, et al. [Health professionals facing the coronavirus disease 2019 (COVID-19) pandemic: What are the mental health risks?]. Encephale. 2020; 46(3s): S73-s80. PMid: 32370984. https://doi.org/10.1016/j.encep. 2020 .04 .008

[6] Abdulah DM, Musa DH. Insomnia and stress of physicians during COVID-19 outbreak. 2020; 2: 100017. https://doi.org/10.1 016/j.sleepx.2020.100017

[7] Cai Q, Feng H, Huang J, et al. The mental health of frontline and non-frontline medical workers during the coronavirus disease 2019 (COVID-19) outbreak in China: A case-control study. J Affect Disord. 2020; 275: 210-5. PMid: 32734910. https ://doi.org/10.1 $016 / \mathrm{j} \cdot \mathrm{jad} .2020 .06 .031$

Published by Sciedu Press
[8] Pappa S, Ntella V, Giannakas T, et al. Prevalence of depression, anxiety, and insomnia among healthcare workers during the COVID-19 pandemic: A systematic review and meta-analysis. Brain Behav Immun. 2020; 88: 901-7. PMid: 32437915. https://doi.org/10.1 016/j.bbi.2020.05.026

[9] Shechter A, Diaz F, Moise N, et al. Psychological distress, coping behaviors, and preferences for support among New York healthcare workers during the COVID-19 pandemic. Gen Hosp Psychiatry. 2020; 66: 1-8. PMid: 32590254. https://doi.org/10.1016/j.genh osppsych.2020.06.007

[10] Khanam A, Dar SA, Wani ZA, et al. Healthcare Providers on the Frontline: A Quantitative Investigation of the Stress and Recent Onset Psychological Impact of Delivering Health Care Services During COVID-19 in Kashmir. Indian Journal of Psychological Medicine. 2020; 42(4): 359-67. PMid: 33402797. https: //doi.org/10.1177/0253717620933985

[11] Galehdar N, Kamran A, Toulabi T, et al. Exploring nurses' experiences of psychological distress during care of patients with COVID19: a qualitative study. BMC Psychiatry. 2020; 20(1): 489. PMid: 33023535. https://doi .org/10.1186/s12888-020-02898-1

[12] Karampelias V, Karonis D, Psaroudi V. The psycho-emotional impact of COVID-19 on surgical staff working in emergency departments. Eur J Trauma Emerg Surg. 2020; 46(4): 747-9. PMid: 32494836. https://doi.org/10.1007/s00068-020-01411-3

[13] Schwartz R, Sinskey JL, Anand U, et al. Addressing Postpandemic Clinician Mental Health: A Narrative Review and Conceptual Framework. Ann Intern Med. 2020. PMid: 32822206. https : //doi.org/10.7326/M20-4199

[14] Albott CS, Wozniak JR, McGlinch BP, et al. Battle Buddies: Rapid Deployment of a Psychological Resilience Intervention for Health Care Workers During the COVID-19 Pandemic. Anesth Analg. 2020; 131(1): 43-54. PMid: 32345861. https://doi .org/10.1213/AN E. 0000000000004912 
[15] Blake H, Bermingham F, Johnson G, et al. Mitigating the Psychological Impact of COVID-19 on Healthcare Workers: A Digital Learning Package. Int J Environ Res Public Health. 2020; 17(9). PMid: 32357424. https://doi.org/10.3390/ijerph17092997

[16] Wu PE, Styra R, Gold WL. Mitigating the psychological effects of COVID-19 on health care workers. Cmaj. 2020; 192(17): E459-e60. PMid: 32295761. https ://doi .org/10.1503/cmaj . 200519

[17] Hall DL, Millstein RA, Luberto CM, et al. Responding to COVID-19 Stress: Disseminating Mind-Body Resiliency Approaches. Global Advances in Health and Medicine. 2020; 9: 2164956120976554. PMid: 33312763. https://doi.org/10.1177/216495612097 6554

[18] Park ER, Sylvia LG, Streck JM, et al. Launching a resiliency group program to assist frontline clinicians in meeting the challenges of the COVID-19 pandemic: Results of a hospital-based systems trial. Gen Hosp Psychiatry. 2020. PMid: 33229013. https: //doi.org/10.1016/j.genhosppsych.2020.10.005
[19] Bradley EH, Curry LA, Devers KJ. Qualitative data analysis for health services research: developing taxonomy, themes, and theory. Health Serv Res. 2007; 42(4): 1758-72. PMid: 17286625. https://doi.org/10.1111/j.1475-6773.2006.00684.x

[20] Braun V, Clarke V. Using thematic analysis in psychology. Qualitative Research in Psychology. 2006; 3(2): 77-101. https://doi .or g/10.1191/1478088706qp063oa

[21] Magill BAE, Siegel Z, Pike KM. The Mental Health of Frontline Health Care Providers During Pandemics: A Rapid Review of the Literature. Psychiatric Services. 2020; 71(12): appips202000274. PMid: 33019857. https://doi.org/10.1176/appi.ps. 202000274

[22] Pollock A, Campbell P, Cheyne J, et al. Interventions to support the resilience and mental health of frontline health and social care professionals during and after a disease outbreak, epidemic or pandemic: a mixed methods systematic review. Cochrane Database Syst Rev. 2020; 11: Cd013779. PMid: 33150970. https://doi .org/10.1 002/14651858.CD013779 